Accepted for publication in ApJ

\title{
The dynamics of the outer parts of $\omega$ Centauri
}

\author{
G. S. Da Costa \\ Research School of Astronomy \& Astrophysics, Australian National University, Mt Stromlo \\ Observatory, via Cotter Rd, Weston, ACT 2611, Australia
}

\begin{abstract}
The multi-object fibre-fed spectrograph AAOmega at the Anglo-Australian Telescope has been used to establish and measure accurate $\left(\leq 1 \mathrm{~km} \mathrm{~s}^{-1}\right)$ radial velocities for a new sample of members in the outer parts of the stellar system $\omega$ Centauri. The new sample more than doubles the number of known members with precise velocities that lie between $25^{\prime}$ and $45^{\prime}$ from the cluster center. Combining this sample with earlier work confirms that the line-of-sight velocity dispersion of $\omega$ Cen remains approximately constant at $\sim 6.5 \mathrm{~km} \mathrm{~s}^{-1}$ in the outer parts of the cluster, which contain only a small fraction of the total cluster stellar mass. It is argued that the approximately constant velocity dispersion in the outer regions is most likely a consequence of external influences, such as the tidal shock heating that occurs each time $\omega$ Cen crosses the Galactic plane. There is therefore no requirement to invoke dark matter or non-standard gravitational theories.
\end{abstract}

Subject headings: globular clusters: general; globular clusters: individual ( $\omega$ Centauri, NGC 5139); stars: kinematics and dynamics

\section{Introduction}

The stellar system $\omega$ Centauri has been known to be unusual, at least as regards its stellar population, for almost four decades. There is now an extensive body of work which shows that, unlike the situation for most globular clusters, the member stars of $\omega$ Cen possess a large range in heavy element abundance together with distinctive element-to-iron abundance ratios (e.g., Johnson \& Pilachowski 2010, and the references therein). A substantial spread in Helium abundance has also been inferred from the observed abundances and structure of the lower main sequence in the cluster color-magnitude diagram (e.g., Norris 2004; Piotto et al. 2005). Investigations of the metallicities of $\omega$ Cen stars in the vicinity of the main sequence turnoff also suggest that the cluster has an age spread of perhaps 2 Gyr (e.g., Pancino et al. 2011, and the references therein). Together these characteristics have led to the suggestion that $\omega$ Cen has not evolved in isolation but is instead the nuclear remnant of a now disrupted nucleated dwarf galaxy that was accreted by the Milky 
Way (e.g., Freeman 1993). Bekki \& Freeman (2003) have shown that despite the tightly bound and retrograde current orbit of $\omega$ Cen, such a disruption and accretion process is dynamically plausible. Nevertheless, the spectroscopic survey of Da Costa \& Coleman (2008) showed that there is little evidence for any significant extra-tidal population surrounding $\omega$ Cen at the present day, consistent with the photometric study of Law et al. (2003). Da Costa \& Coleman (2008, hereafter DC08) give an upper limit of $0.7 \%$ for the fraction of the cluster mass contained between 1 and 2 cluster tidal radii. This result requires the tidal stripping and disruption process of the postulated progenitor system to be largely complete at early epochs with the stars from the disrupted dwarf galaxy now widely distributed around the Galaxy (e.g., Wylie-de Boer et al. 2010; Majewski et al. 2012).

While the nucleosynthetic history of $\omega$ Cen is complicated and not fully understood, the dynamics of the present-day stellar system, at least for the part of the cluster containing most of the stellar mass, are relatively well established. There have been a number of models of the system including those of Mevlan (1987), Mevlan et al. (1995), Merritt et al. (1997), Giersz \& Heggie (2003) and van de Marel \& Anderson (2010), all of which, within their adopted assumptions, reproduce well the available observational data. The most detailed model is that of van de Ven et al. (2006). This axisymmetric dynamical model, which includes rotation and radially varying anisotropy, suggests that the mass-to-light ratio of $\omega$ Cen does not change with radius - the variation in the model $M / L_{V}$ value does not deviate significantly from the best-fit constant value of 2.5 (solar units) out to the limits of the modelled data at $r \sim 20^{\prime}$ (van de Ven et al. 2006).

However, it is necessary to keep in mind that models such as that of van de Ven et al. (2006) are constrained by the extent of available observational data. In the case of the velocity dispersion profile for $\omega$ Cen, the data have been limited, until relatively recently, to a radius of approximately $20^{\prime}$ from the cluster center. While this radius ( $\sim 4$ half-light radii) contains most of the cluster stellar mass, it nevertheless is less than half the nominal "tidal radius" of $\omega$ Cen ( $57^{\prime}$; see the discussion in DC08). The lack of information on the velocity dispersion profile at large radii may mean we are currently missing some interesting astrophysics. For example, if $\omega$ Cen is the nuclear remnant of a disrupted dwarf galaxy then it is possible it has retained some of the dark matter content of the original system. One of the best places to constrain the dark matter content is in the outer parts of the cluster where the stellar densities are low (e.g., Carraro \& Lia 2000; Mashchenko \& Sills 2005).

Scarpa et al. (2003) presented the first data for the line-of-sight velocity dispersion of $\omega$ Cen beyond $\sim 20^{\prime}$ from the cluster center. They used accurate radial velocities for 75 members with $\sim 20$ $\leq r^{\prime} \leq 30$ to show that the cluster velocity dispersion profile may be relatively flat beyond $20^{\prime}$. This is in contrast to the monotonically declining dispersion profile expected for a system in dynamical equilibrium in which mass follows light. Scarpa et al. (2003) chose to interpret their results as indicating the breakdown of Newtonian dynamics in a weak acceleration regime. However, this interpretation has been questioned by, for example, Baumgardt et al. (2005) who argue that the external influence of the Milky Way on clusters such as $\omega$ Cen, that lie relatively close the Galactic Center, is sufficiently large that the effective acceleration is larger than the critical MOND constant $a_{0}$; thus Newtonian dynamics should still apply. 
Two additional studies of the velocity dispersion in the outskirts of $\omega$ Cen have recently appeared. In the first, Sollima et al. (2009) conducted a survey for new $\omega$ Cen members in the outer regions of the cluster and combined their radial velocity results with those from the earlier study of Pancino et al. (2007) to generate a velocity dispersion profile for the cluster that reached a radial distance of $\sim 32^{\prime}$. The typical uncertainty in the velocity dispersion measures was $\leq 1$ $\mathrm{km} \mathrm{s}^{-1}$. Sollima et al. (2009) claim that the velocity profile decreases monotonically from the center outwards though their outermost data point lies above the previous point by more than the combined $(1 \sigma)$ errors. Sollima et al. (2009) note that this occurence might be compatible with the onset of tidal heating in the outskirts of the cluster. Nevertheless, the Sollima et al. (2009) data are not inconsistent with a constant velocity dispersion beyond $r \approx 20^{\prime}$. The Sollima et al. (2009) sample contains $98 \omega$ Cen members beyond $r \approx 20^{\prime}$ but of these stars only 13 lie beyond $30^{\prime}$.

The second recent paper is that of Scarpa \& Falomo (2010) in which the data sets of Sollima et al. (2009) and Scarpa et al. (2003) have been combined to provide a further estimate of the velocity dispersion profile. The addition of the Scarpa et al. (2003) velocities increases the number of stars with accurate velocities for radial distances between $20^{\prime}$ and $30^{\prime}$ but does not contribute any new members beyond $r \approx 30^{\prime}$. The combined data are consistent with a flattening of the velocity dispersion beyond $r \approx 20^{\prime}$. Scarpa \& Falomo (2010) conclude that this dispersion profile clearly deviates from the "Newtonian prediction", by which they mean a monotonically declining dispersion profile, and is "best explained by a breakdown of Newtonian dynamics below a critical acceleration".

Clearly there is an urgent need for additional accurate radial velocities for bona-fide members of $\omega$ Cen in the outskirts of the cluster, particularly beyond $r \approx 30^{\prime}$. The generation of such a sample and a redetermination of the line-of-sight velocity dispersion profile in the outer regions of $\omega$ Cen is the purpose of this paper. The sample selection, the observations, and the measurement of the radial velocities are discussed in the next section. Section 3 discusses the membership status of the candidates, which is important given the low density of $\omega$ Cen members in the outer regions of the cluster. Section 4 presents the velocity dispersion profiles derived from both the new observations and from combining the new data with the velocities given by Sollima et al. (2009) and Scarpa \& Falomo (2010). The results are presented and discussed in $\S 5$.

\section{Observations and Reductions}

\subsection{Sample Selection}

The list of candidates to be observed consisted first of the 154 probable $\omega$ Cen members identified in DC08. These stars lie between $20^{\prime}$ and $55^{\prime}$ from the cluster center, possess velocities and line strengths consistent with cluster membership, and have $15.4 \leq V \leq 16.75$ (DC08). Then, in order to increase the sample of potential members in the outer parts of the cluster, two further lists were generated. The first was simply the stars in the original DC08 sample that lie between $30^{\prime}$ and $60^{\prime}$ from the cluster center and which were not observed in DC08. There are 545 candidates 
in this category. Note that the outer radius limit of $60^{\prime}$ was chosen to match with the 2 degree diameter field-of-view of the fiber-positioning system at the prime focus of the AAT, enabling fiber configurations to be centered on the cluster. It also matches the "tidal radius" of $\omega$ Cen.

The second candidate list was generated from the same photometry set as used in DC08 but the selection window was extended $\sim 0.5$ mag fainter parallel to the cluster sequence in the colormagnitude diagram (see Fig. 1 of DC08). As for the brighter sample, the selected stars lie between $30^{\prime}$ and $60^{\prime}$ from the cluster center. There are 1798 additional candidates is this list.

\subsection{Observations}

Five nights in 2008 April were allocated to this program with the $2 \mathrm{dF}$ fibre positioner and the AAOmega spectrograph on the $3.9 \mathrm{~m}$ Anglo-Australian Telescope (AAT). The fibre positioner at the AAT prime focus can allocate a maximum of 392 fibers within the 2 degree diameter field-ofview. Each fibre configuration then consisted of 6-8 guide fibre bundles, $\sim 300$ fibres allocated to candidate members, up to 50 fibres allocated to blank-sky and $\sim 20$ fibres to likely members from DC08. These latter stars were incorporated into every configuration to monitor for any systematic effects in the radial velocities. The fibres from the positioner are fed to the AAOmega spectrograph - a double beam instrument with separate blue and red cameras (Saunders et al. 2004; Sharp et al. 2006). The spectrograph was configured with the $\lambda 5700 \AA$ dichroic and the $1500 \mathrm{~V}$ (blue) and 1700D gratings (red). The blue spectra cover the wavelength interval $\lambda \lambda 4940-5650 \AA$ at a resolution $\lambda / \Delta \lambda$ of $\sim 4000$. The red spectra were centred at $\lambda 8600 \AA$ with coverage from $\lambda 8340 \AA$ to $\lambda 8775 \AA$ including the Ca II triplet lines at $\lambda 8498,8542$ and $8662 \AA$. The resolution is $\sim 10,000$ and the scale corresponds to $8.5 \mathrm{~km} \mathrm{~s}^{-1}$ per pixel. In this paper we concentrate on the red camera data only.

Less than ideal weather meant that only $11 \omega$ Cen candidate member fibre configurations were observed, principally on 2008 April 25 and 2008 April 26. Two of these configurations were repeats to compensate for diminished signal due to cirrus affecting earlier observations. Each configuration was observed as a set of $3 \times 1500$ sec exposures preceded by fibre-flat and arc lamp exposures and followed by a second arc lamp exposure. A number of bright radial velocity standards were also observed through individual fibres during the run. These provide template spectra for the crosscorrelation analysis used to determine radial velocities. Each dataset was reduced with the pipeline reduction code $2 d f d \sqrt{1}$ which generates a wavelength-calibrated sky-subtracted spectrum for each object fibre. The relative fibre transmissions were set from the data using the SKYLINE(MED) option which makes use of the significant flux in each raw spectrum from the numerous bright night-sky emission lines in the wavelength region covered by the red camera data. The individual spectra from each integration were then median combined to remove cosmic-ray contamination.

An additional configuration made up of likely new members as determined from the April

\footnotetext{
${ }^{1}$ www.aao.gov.au/AAO/2df/aaomega/aaomega_software.html \#drcontrol
} 
observations was observed on 2008 May 31 via the AAT Service Observing program. The instrumental set-up for the service observations on the red side of AAOmega was the same as for the April observations and the data were observed and reduced in an identical fashion.

\subsection{Radial Velocities}

Radial velocities were determined by cross-correlation techniques using the IRAF routine $f x$ cor. The template was a high signal-to-noise spectrum of the $V=7.7 \mathrm{~F} 6 \mathrm{~V}$ star HD160043, which provides a good match to the $\omega$ Cen member spectra, particularly as regards the width and depth of the Ca II triplet lines. The wavelength interval used for the correlation was $\lambda \lambda 8470-8740 \AA$ which encompasses the Ca II triplet lines as well as a number of weaker lines, while minimising regions of potential significant residual from the sky subtraction. After the cross-correlations were computed, the output velocity error and the cross-correlation peak height were plotted against the continuum count level in the correlation wavelength region for each of the 12 observed configurations. This enabled the identification of occasional situations where the correlation had been affected by instrumental effects such as inadequate cosmic-ray removal. In such cases the problem was corrected, usually by interpolating over the effected pixels, and the cross-correlation repeated. In the final analysis velocities that had output errors exceeding $5 \mathrm{~km} \mathrm{~s}^{-1}$ and/or cross-correlation peak heights less than 0.5 were discarded - these always coincided with the lowest signal-to-noise spectra.

After applying appropriate heliocentric corrections, the zero point of the velocity system was determined by correlating the template spectrum with other observations of the same standard in different fibres and with similar spectra of three other radial velocity standards (HD83516, HD101266 and HD162356). The zero point was set by minimising the difference between the observed relative velocities for these stars and their catalogue values. The 11 observations of the four standards then have a standard deviation of $0.8 \mathrm{~km} \mathrm{~s}^{-1}$ about their catalogue values indicating the velocity zero point is well determined. Only the 245 stars with velocities exceeding $100 \mathrm{~km} \mathrm{~s}^{-1}$ were then retained for the subsequent analysis.

To determine the velocity errors the rms deviation about the mean velocity was first calculated for the $\sim 20 \omega$ Cen members observed in the majority, if not all, of the 12 configurations. Both the mean velocity and the rms were calculated using the output velocity errors from fxcor as weights. These data show that the rms about the mean velocity is below $1 \mathrm{~km} \mathrm{~s}^{-1}$ when the continuum level in the cross-correlation region exceeds $\sim 900$ ADU. The rms values then rise relatively rapidly with decreasing continuum levels to $1.7 \pm 0.3(1 \sigma) \mathrm{km} \mathrm{s}^{-1}$ at $\sim 600$ ADU. A similar analysis of the stars observed in the two repeated configurations is consistent with these values and indicates further that the rms continues to rise with decreasing continuum level to $\sim 3 \pm 0.7(1 \sigma) \mathrm{km} \mathrm{s}^{-1}$ at $\sim 200 \mathrm{ADU}$, the lowest continuum level of the stars remaining in the data set. For stars with a single observation the velocity error was then set by this (rms, continuum level) relation while for stars with multiple observations the error was taken as the rms divided by the square-root of the number of observations. The overall median velocity error is less than $1 \mathrm{~km} \mathrm{~s}^{-1}$ excluding any 
systematic zero point uncertainty. This velocity error is lower than the $2-3 \mathrm{~km} \mathrm{~s}^{-1}$ velocity error listed by Lane et al. (2009) who used a similar observing setup, although Lane et al. (2009) do not give any information on the continuum levels of their spectra. The uncertainty in the velocity errors is sufficiently small that its contribution to the uncertainty in the calculated velocity dispersion is negligible.

\section{3. $\omega$ Cen Membership}

In Fig. 1]we plot the radial velocity, corrected for perspective rotation (see Sollima et al. 2009; van de Ven et al. 2006) against distance from the cluster center in arcmin. The radial distances are computed using a tangent plane projection (see Sollima et al. 2009; van de Ven et al. 2006) to allow for the large angular diameter of the field surveyed. Considering first the 109 stars from DC08 with radial distances between $20^{\prime}$ and $30^{\prime}$, it is evident that the vast majority of these stars are apparently probable cluster members despite the relatively low velocity precision $\left(\sim 11 \mathrm{~km} \mathrm{~s}^{-1}\right)$ of the earlier study. Only one star, 8_7_16862, is definitely reclassified as a non-member based on the velocity determined here of $278.8 \mathrm{~km} \mathrm{~s}^{-1}$, while a second star, 8_3_1066 with $\mathrm{v}_{r}=254.2 \mathrm{~km}$ $\mathrm{s}^{-1}$, lies just outside the $\pm 20 \mathrm{~km} \mathrm{~s}^{-1}$ from the cluster mean boundaries shown in the figure. This star is retained as a possible member for the moment.

For the 136 stars with $\mathrm{v}_{r} \geq 100 \mathrm{~km} \mathrm{~s}^{-1}$ and which lie beyond $30^{\prime}$ from the cluster center, Fig. 1 shows that there is an apparent grouping around the cluster mean velocity out to a radius of at least $45^{\prime}$ and possibly beyond. However, it must be kept in mind that the surface density profile of the cluster is dropping rapidly with increasing radius: the profile given in DC08 indicates that the cluster star density drops by a factor of 5 between $20^{\prime}$ and $30^{\prime}$ and by a further factor of 10 between $30^{\prime}$ and $40^{\prime}$. Conversely, the area that needs to be surveyed goes up as $r^{2}$, as does the number of contaminating non-members assuming they have a uniform surface density. For these reasons coincidence with the $\omega$ Cen mean velocity does not guarantee cluster membership in the outer regions of the cluster, and additional information must be used to help exclude non-members. Fortunately, we can make use of the known properties of the stellar population of $\omega$ Cen to carry out this task.

The stellar system $\omega$ Cen is well known for its internal spread in $[\mathrm{Fe} / \mathrm{H}]$ abundance. Johnson \& Pilachowski (2010) have provided $[\mathrm{Fe} / \mathrm{H}]$ values for a large sample of $\omega$ Cen red giants and their results show, in agreement with earlier work (see Johnson \& Pilachowski 2010, for references), that: (a) there is a lower bound at $[\mathrm{Fe} / \mathrm{H}] \approx-1.9$ to the abundances of $\omega$ Cen red giants that remains constant with increasing radius; (b) there is a decrease in the number of stars with $[\mathrm{Fe} / \mathrm{H}] \geq-1.3$ with increasing radius relative to the number of more metal-poor stars; and (c) the dispersion in abundance for stars with $[\mathrm{Fe} / \mathrm{H}] \leq-1.3$ is approximately constant with radius. The Johnson \& Pilachowski (2010) sample reaches out to radial distances of only $\sim 24^{\prime}$ but the limited sample of more distant stars in Norris et al. (1997) suggest that these results apply also at larger radii. Further, despite the complexity of the $\omega$ Cen color-magnitude diagram (CMD) in the vicinity of the main sequence 


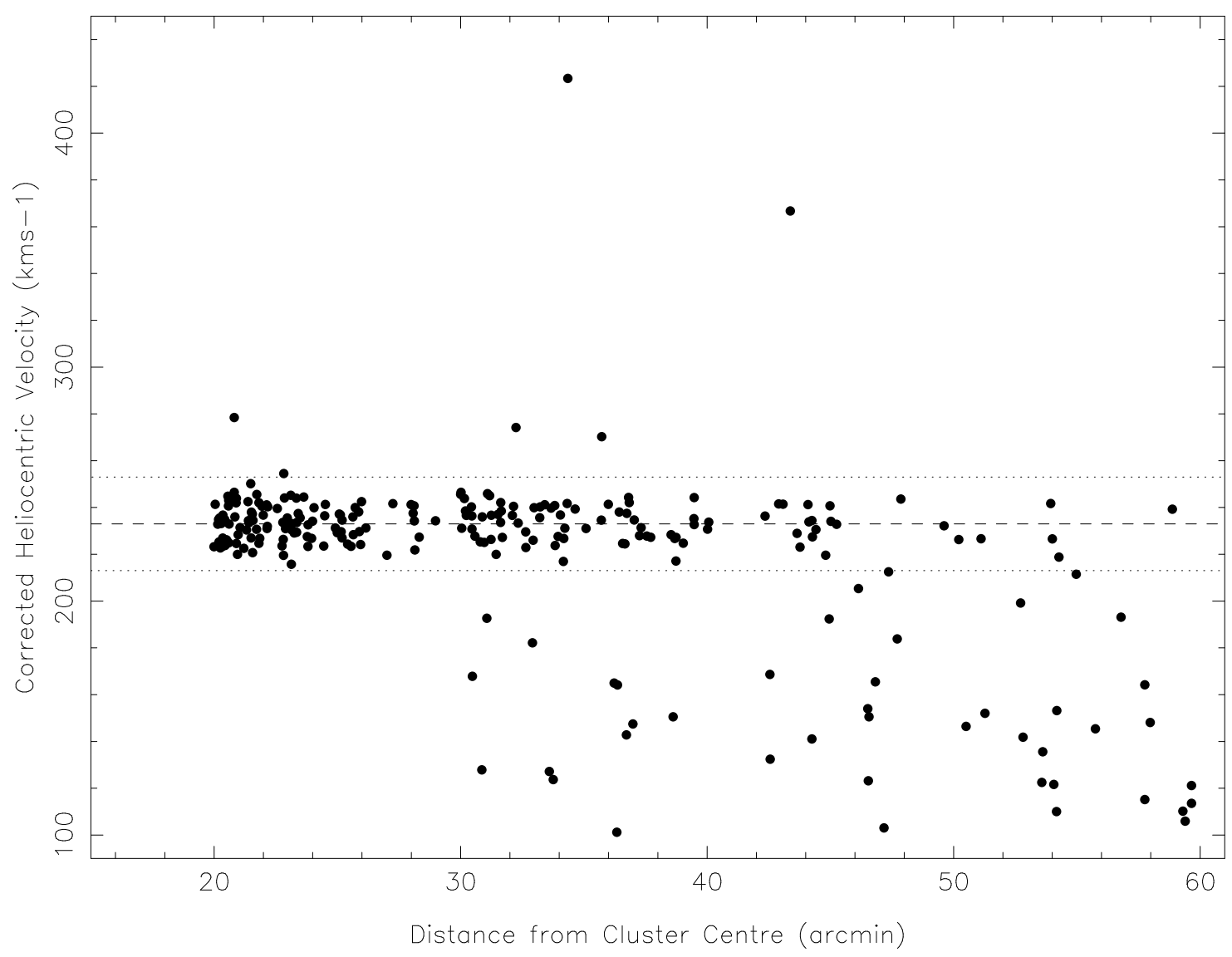

Fig. 1. - The radial velocity, corrected for perspective rotation, is plotted against distance from the center of $\omega$ Cen for all stars observed whose velocities exceed $100 \mathrm{~km} \mathrm{~s}^{-1}$. Stars between $20^{\prime}$ and $30^{\prime}$ come from the sample of probable members of Da Costa \& Coleman (2008) while the stars at larger radii includes stars from that sample as well as stars observed here for the first time. The dashed line is at the cluster mean velocity of $233 \mathrm{~km} \mathrm{~s}^{-1}$ and the dotted lines are at $\pm 20 \mathrm{~km} \mathrm{~s}^{-1}$ from the mean. 
turnoff (e.g. Bellini et al. 2010), it is likely that the age-range among the cluster stars is comparatively small, less than 2-4 Gyr (e.g., Pancino et al. 2011, and the references therein). Thus there should be a reasonable level of consistency between overall abundance inferred from location on the red giant branch in the CMD and that inferred spectroscopically. We can therefore use the photometry and the line strengths for the stars in the velocity window $213-253 \mathrm{~km} \mathrm{~s}^{-1}$ to select probable members at all radial distances.

The approach is as follows. In Fig. 2 we show in the upper panel the CMD for the 108 stars in the velocity range $213-253 \mathrm{~km} \mathrm{~s}^{-1}$ (plus 8_3_1066) which have radial distances between $20^{\prime}$ and $30^{\prime}$. The lower panel shows the CMD for stars in the same velocity range but with radial distances between $30^{\prime}$ and $60^{\prime}$. These two groupings are designated the inner and outer samples, respectively. Note that for completeness we have included in the outer sample stars 5_3_226 and 9_4_1918 as with velocities of $212.5 \pm 1.2$ and $211.4 \pm 1.5 \mathrm{~km} \mathrm{~s}^{-1}$, respectively, they lie very close to the lower limit of the velocity selection range (see Fig. 1). Shown also in both panels are theoretical isochrones from the Dartmouth Stellar Evolution Database (Dotter et al. 2008) for an age of 13 Gyr and metallicities $[\mathrm{Fe} / \mathrm{H}]$ of $-2.0,-1.5,-1.0$ and -0.5 dex, respectively. For the three lower metallicities the isochrones are for $[\alpha / \mathrm{Fe}]=+0.4$ while the most metal-rich isochrone has $[\alpha / \mathrm{Fe}]=+0.2$ dex. This is consistent with the dependence of $[\alpha / \mathrm{Fe}]$ on $[\mathrm{Fe} / \mathrm{H}]$ in the cluster (e.g., Pancino et al. 2002; Johnson \& Pilachowski 2010). The isochrones have been fitted assuming $(\mathrm{m}-\mathrm{M})_{V}=13.94$ and $\mathrm{E}(V-I)=0.16 \mathrm{mag}(\mathrm{E}(B-V)=0.12 \mathrm{mag})$ as tabulated in the 2010 version of the Milky Way Globular Cluster database (Harris 1996, hereafter H10).

Similarly we show in Fig. 3 plots of the combined equivalent widths of the $\lambda 8542 \AA$ and $\lambda 8662 \AA$ lines of the Ca II triplet against $V-V_{H B}$ and $V-I$ for the inner and outer samples. The equivalent widths have been determined using gaussian fits to the line profiles with feature and continuum bandpasses similar to those adopted in the original work of Armandroff \& Da Costa (1991). The $V_{H B}$ value for $\omega$ Cen was taken from H10. The values of $V-V_{H B}$ lie outside the range of existing abundance calibrations for the Ca II triplet which are tailored to more luminous red giants. Nevertheless we can use the inner sample, which is dominated by cluster members, to define reasonable upper and lower envelopes for the relation between line strength and $V-V_{H B}$ followed by $\omega$ Cen stars. The adopted linear relations are shown as the dashed lines in the upper left panel of Fig. 3. The relations are then duplicated for the outer sample as shown in the upper right panel of the figure. In both cases the lower envelope is very well defined consistent with the result for more luminous samples that the lower abundance cutoff in the abundance distribution is quite sharp (e.g., Norris et al. 1996; Johnson \& Pilachowski 2010). The upper envelope is less well defined, as expected, given the significant range of metallicities present even at large radial distances (Norris et al. 1997). The lower panels of Fig. 3 show the relation between the Ca II triplet strength and $(V-I)$ colour for the two samples. Members of $\omega$ Cen should show a broad correlation between these quantities with, in general, stronger lines going with redder colours. The panels show this is the case.

We then combine the information in Figs. 2 and 3 as follows. For each star in the panels of 


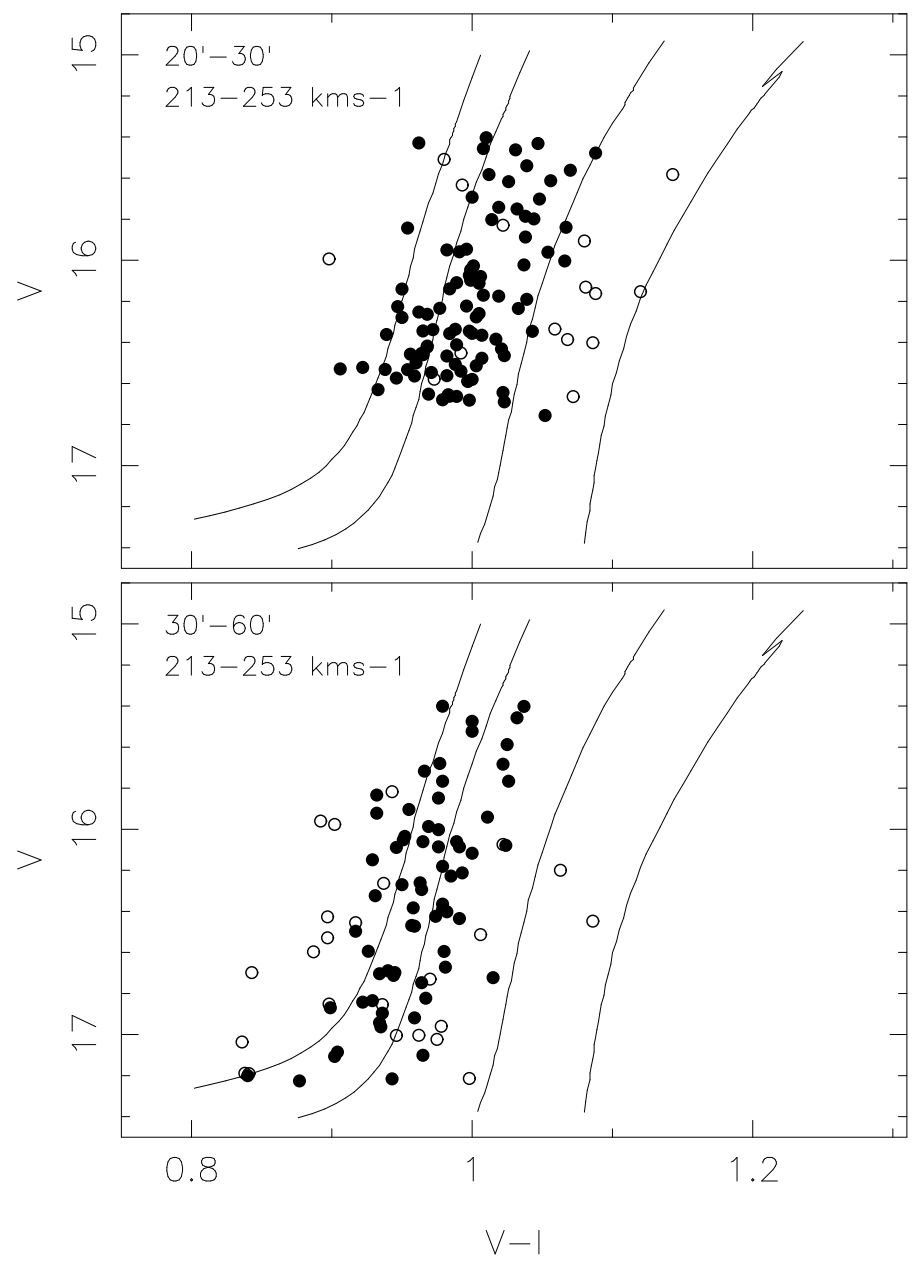

Fig. 2.- Color-Magnitude Diagrams for stars with radial velocities between 213 and $253 \mathrm{~km} \mathrm{~s}^{-1}$ and distances from the cluster center of $20^{\prime}-30^{\prime}$ (upper panel) and $30^{\prime}-60^{\prime}$ (lower panel). Shown also are Dartmouth isochrones for an age of $13 \mathrm{Gyr}$ and (left-to-right) $([\mathrm{Fe} / \mathrm{H}],[\alpha / \mathrm{Fe}])$ values of $(-2.0,+0.4),(-1.5,+0.4),(-1.0,+0.4)$, and $(-0.5,+0.2)$, respectively. The isochrones have been fitted assuming $(\mathrm{m}-\mathrm{M})_{V}=13.94$ and $\mathrm{E}(V-I)=0.16$ mag. The stars adopted as cluster members are plotted as filled symbols while the likely non-members are plotted as open symbols. 

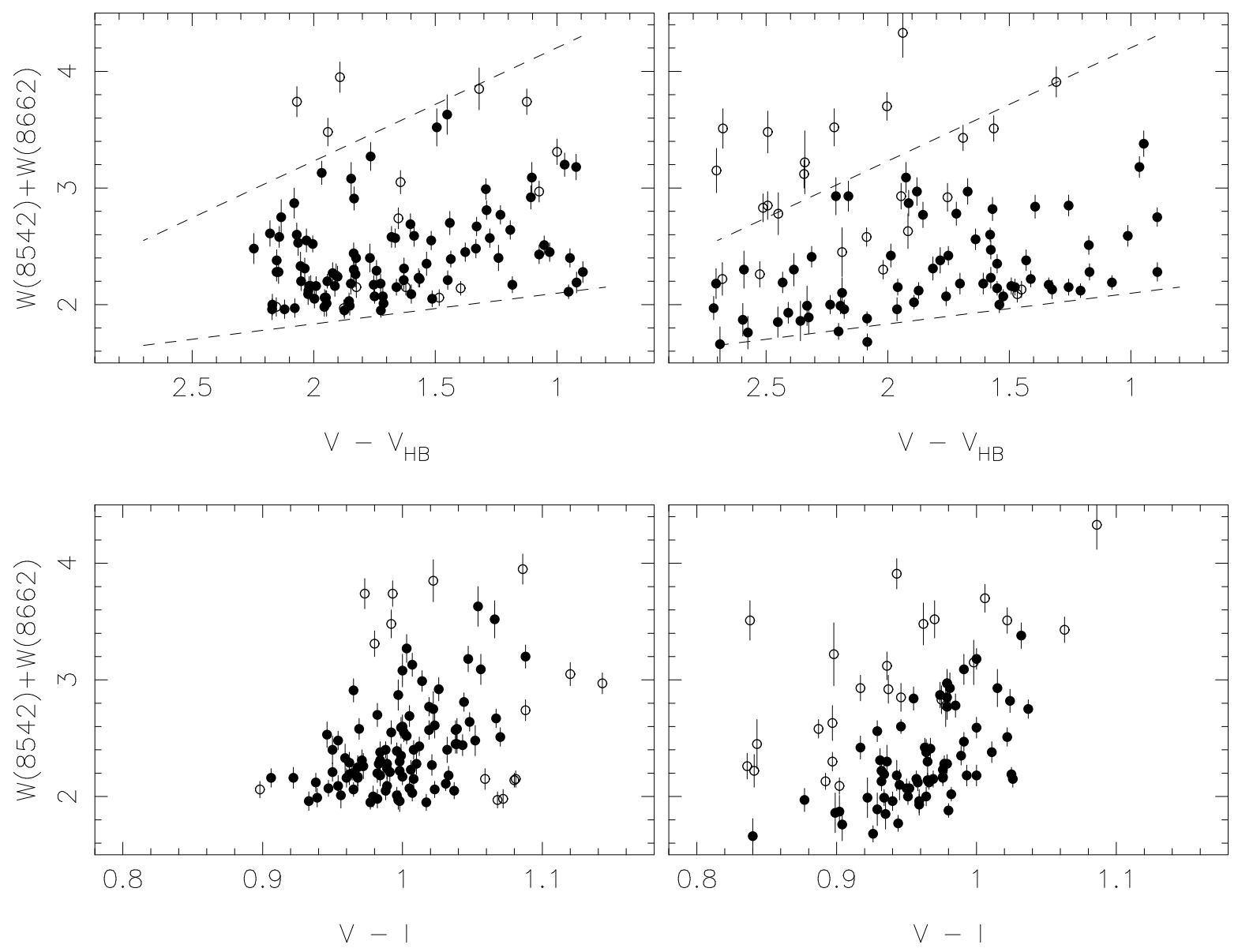

Fig. 3. - The sum of the equivalent widths in $\AA$ of the Ca II triplet lines at $\lambda 8542 \AA$ and $\lambda 8662 \AA$ lines is plotted against $V-V_{H B}$ (upper panels) and $V-I$ (lower panels) for the inner sample (left panels) and the outer sample (right panels). The linear segments in the upper left panel encompass the range of line strengths shown by probable cluster members in the inner sample. The segments are replicated in the upper right panel. The stars adopted as cluster members are plotted as filled symbols while the likely non-members are plotted as open symbols. 
Fig. 2 we measure the offset between the $V-I$ color of the star and the color of the $13 \mathrm{Gyr},[\mathrm{Fe} / \mathrm{H}]$ $=-2.0,[\alpha / \mathrm{Fe}]=+0.4$ isochrone at the star's $V$ magnitude, normalized by the color difference between this isochrone and that for $13 \mathrm{Gyr},[\mathrm{Fe} / \mathrm{H}]=-0.5,[\alpha / \mathrm{Fe}]=+0.2$ at the $V$ mag of the star. For the same star we then measure the difference in equivalent width between the value for the star and the lower envelope line shown in the upper panels of Fig. 3 at the $V-V_{H B}$ value for the star. This difference is then normalized by the equivalent width difference between the upper and lower lines at the $V-V_{H B}$ value of the star. For members of the cluster these two quantities, denoted by $\delta_{n}(\Sigma \mathrm{W})$ and $\delta_{n}(V-I)$ respectively, should be well correlated as both are metallicity indicators. Non-members, however, will in general lie away from the cluster member sequence. The results of this process are shown in Fig. 4 where the expected correlations are evident. The dashed lines in the upper panel of the figure show the membership selection window adopted for the inner sample. The selection window is then reproduced in the lower panel for the outer sample.

We have then combined the information from Figs. 2, 3 and 4 to provide our best estimate of the $\omega$ Cen membership status for the stars in the inner and outer samples. The adopted cluster members are plotted as filled symbols in all three figures while the non-members are plotted as open symbols. We note that the membership status of stars falling near the boundaries of the selection window in Fig. 4 were individually considered and classified taking into consideration uncertainties in the photometry and line strength measurements. In particular, despite our efforts to minimise them it is still likely that there are systematic uncertainties in the $(V-I)$ photometry at the \pm 0.03 mag level. For inner sample, 93 of the original 108 stars are classified as members, including star 8_3_1066, while for the outer sample, 67 of the original 91 are classified as members, including 5_3_226 but not 9_4_1918. We then list in Table 1 the identification, J2000 position, heliocentric velocity and error, distance from the cluster center in arcmin, $V$ and $V-I$ photometry, and the sum of the equivalent widths of the $\lambda 8542 \AA$ and $\lambda 8662 \AA$ Ca II triplet lines in $\AA$, together with its associated error, for the 160 adopted cluster members. Table 2 gives the same information for 39 stars from the inner and outer samples that are classified as probable non-members of $\omega$ Cen. Figure 5 shows the observed velocity versus radial distance diagram of Fig. 1 but now with the probable members and non-members identified.

We have then used the Besancon model of the Galaxy (Robin et al. 2003) to estimate the success of this membership selection process. Five independent realisations of the model Galaxy were generated for the line-of-sight towards $\omega$ Cen using an area on the sky equivalent to that of the $2 \mathrm{dF}$ field-of-view. The $V$ and $V-I$ magnitude and color ranges for the model output were chosen to match approximately those of the outer sample of stars (see the lower panel of Fig. 2). Normalisation of the model to the observational data was then set by the ratio of the number of stars in the outer sample with velocities in the range $140-190 \mathrm{~km} \mathrm{~s}^{-1}$ to the number of model stars in the same velocity interval. The predicted number of field stars in the velocity interval 213-253 $\mathrm{km} \mathrm{s}^{-1}$ could then be calculated from the model numbers. The predicted number of field stars with velocities exceeding $260 \mathrm{~km} \mathrm{~s}^{-1}$ was also calculated as a check. We find that the model normalised in this way over predicts the number of high velocity stars: $8 \pm 1$ stars are predicted versus the 4 


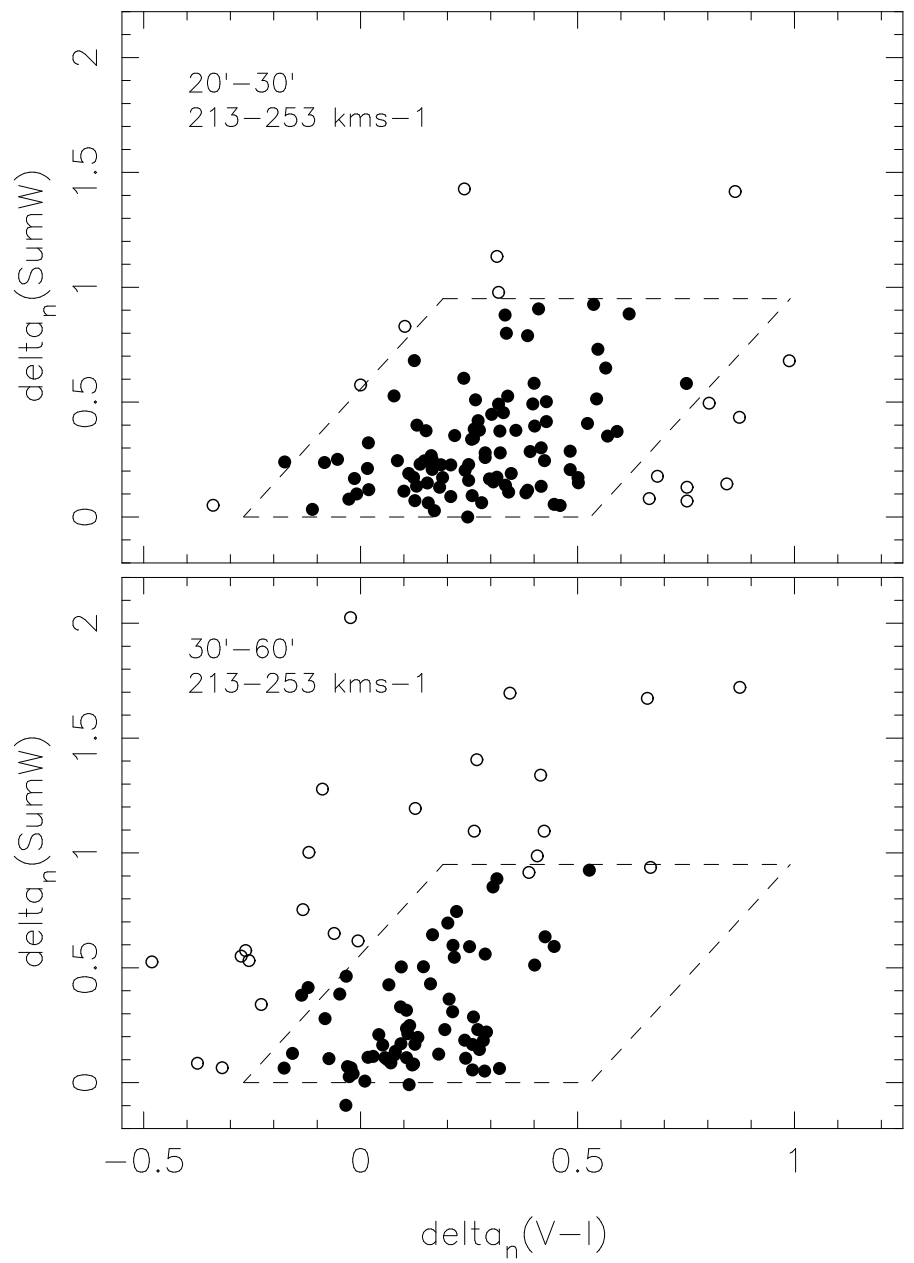

Fig. 4. - The normalized relative line strength $\delta_{n} \Sigma \mathrm{W}$ in $\AA$ is plotted against normalized relative giant branch color $\delta_{n}(V-I)$ for the inner sample (upper panel) and for the outer sample (lower panel). The dashed lines in the upper panel outline the membership selection criteria adopted for the inner sample. The lines are reproduced in the lower panel. The stars adopted as cluster members are plotted as filled symbols while the likely non-members are plotted as open symbols. Stars near the boundaries were considered individually and classified taking into consideration uncertainties in the photometry and line strength measurements. 
Table 1. $\omega$ Cen Probable Member Data

\begin{tabular}{lccccccccc}
\hline \hline \multicolumn{1}{c}{ ID } & RA (2000) & Dec (2000) & $\begin{array}{c}\mathrm{V}_{r} \\
\left(\mathrm{~km} \mathrm{~s}^{-1}\right)\end{array}$ & $\begin{array}{c}\sigma\left(\mathrm{V}_{r}\right) \\
\left(\mathrm{km} \mathrm{s}^{-1}\right)\end{array}$ & $\mathrm{r}^{\prime}$ & $\begin{array}{c}V \\
(\mathrm{mag})\end{array}$ & $\begin{array}{c}V-I \\
(\mathrm{mag})\end{array}$ & $\begin{array}{c}\Sigma \mathrm{W} \\
(\AA)\end{array}$ & $\begin{array}{c}\epsilon \\
(\AA)\end{array}$ \\
\hline 8_7_15831 & 132506.54 & -471741.6 & 223.4 & 0.4 & 20.0 & 15.80 & 1.01 & 2.99 & 0.09 \\
8_4_3206 & 132747.33 & -474544.2 & 241.6 & 1.0 & 20.1 & 16.42 & 0.97 & 2.25 & 0.10 \\
8_8_3134 & 132547.75 & -471059.8 & 232.6 & 0.7 & 20.2 & 16.69 & 1.02 & 2.61 & 0.11 \\
8_6_16385 & 132456.26 & -473634.7 & 235.7 & 0.6 & 20.2 & 16.38 & 1.02 & 1.95 & 0.07 \\
8_2_1336 & 132843.30 & -472437.8 & 234.8 & 0.4 & 20.2 & 16.19 & 1.04 & 2.58 & 0.09 \\
\hline
\end{tabular}

Note. - This table is available in its entirety in a machine-readable form in the online journal. A portion is shown here for guidance regarding its form and content. For stars in common these data supercede those in Table 2 of Da Costa \& Coleman (2008).

Table 2. $\quad \omega$ Cen Probable Non-Member Data

\begin{tabular}{cccccccccc}
\hline \hline ID & RA (2000) & Dec (2000) & $\begin{array}{c}\mathrm{V}_{r} \\
\left(\mathrm{~km} \mathrm{~s}^{-1}\right)\end{array}$ & $\begin{array}{c}\sigma\left(\mathrm{V}_{r}\right) \\
\left(\mathrm{km} \mathrm{s}^{-1}\right)\end{array}$ & $\mathrm{r}^{\prime}$ & $\begin{array}{c}V \\
(\mathrm{mag})\end{array}$ & $\begin{array}{c}V-I \\
(\mathrm{mag})\end{array}$ & $\begin{array}{c}\Sigma \mathrm{W} \\
(\AA)\end{array}$ & $\begin{array}{c}\epsilon \\
(\AA)\end{array}$ \\
\hline 8_8_2219 & 132604.35 & -470939.7 & 224.8 & 0.7 & 20.2 & 16.40 & 1.09 & 3.95 & 0.13 \\
8_8_4146 & 132524.37 & -471335.6 & 226.8 & 0.8 & 20.4 & 16.15 & 1.12 & 3.05 & 0.10 \\
8_1_2780 & 132751.11 & -471128.5 & 235.7 & 0.3 & 20.4 & 15.83 & 1.02 & 3.85 & 0.18 \\
8_8_4052 & 132526.75 & -471255.7 & 240.5 & 0.4 & 20.6 & 16.13 & 1.08 & 2.15 & 0.07 \\
7_3_412 & 132442.89 & -473051.8 & 244.6 & 0.4 & 20.9 & 15.99 & 0.90 & 2.06 & 0.07 \\
\hline
\end{tabular}

Note. - This table is available in its entirety in a machine-readable form in the online journal. A portion is shown here for guidance regarding its form and content. For stars in common these data supercede those in Table 2 of Da Costa \& Coleman (2008). 


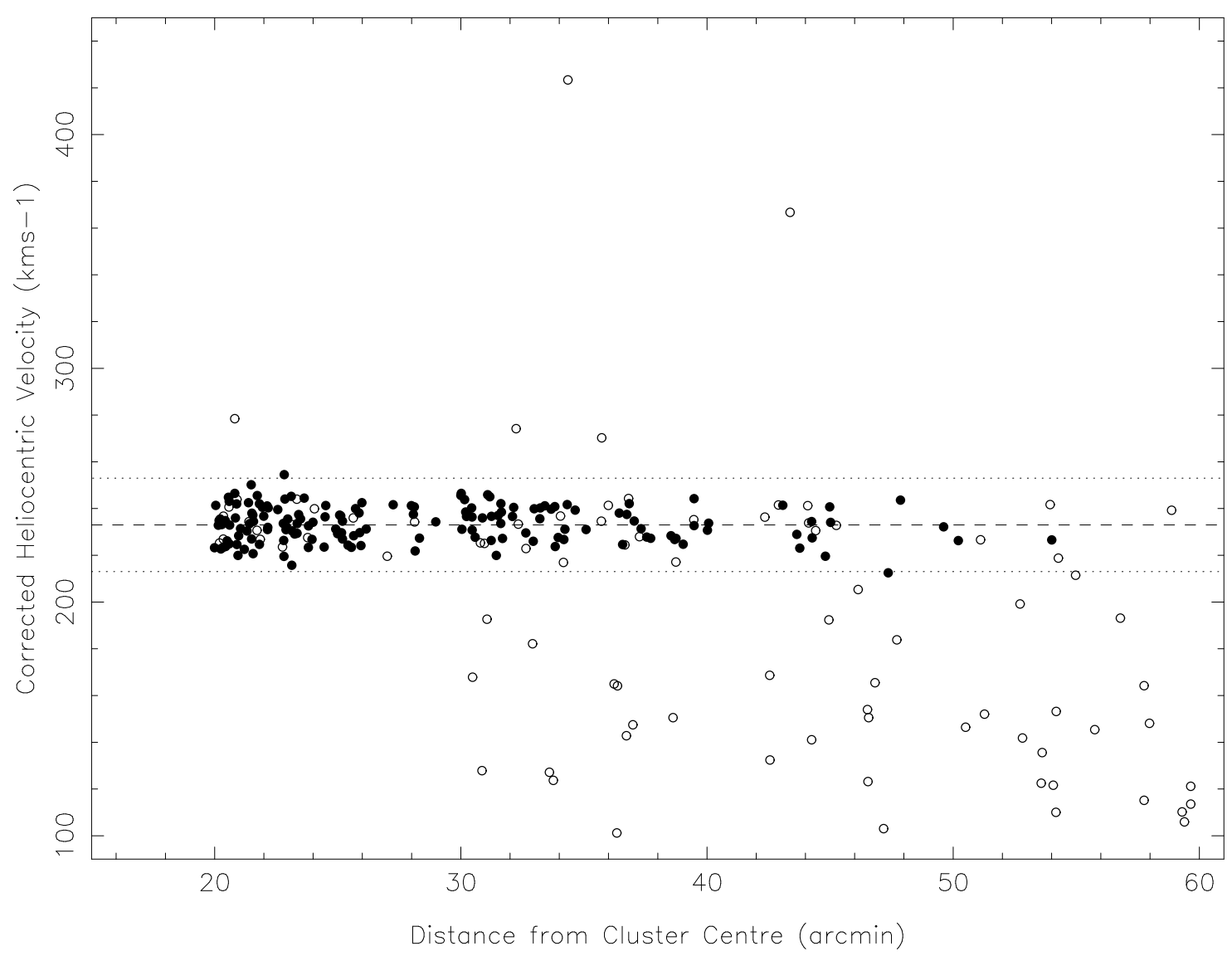

Fig. 5.- As for Fig. 1 the radial velocity, corrected for perspective rotation, is plotted against distance from the center of $\omega$ Cen for all stars observed whose velocities exceed $100 \mathrm{~km} \mathrm{~s}^{-1}$. Adopted probable members are now plotted as filled symbols while probable non-members are plotted as open symbols. 
actually observed in the outer sample. The difference however, is not very significant given the small numbers.

For the cluster velocity range, the normalised model predictions are $3 \pm 1$ field stars for distances from the cluster center between $30^{\prime}$ and $40^{\prime}, 2 \pm 1$ field stars for between $40^{\prime}$ and $50^{\prime}$, and $3 \pm 1$ field stars for between $50^{\prime}$ and $1^{\circ}$. The number of stars classified as probable non-members in the corresponding radial distance intervals (see Fig. 5) are 13, 6 and 4 stars, respectively. Assuming the validity of the model and the normalisation, the comparison then appears to indicate that the membership classification process adopted has in all likelihood been too conservative: perhaps as many as dozen of the "probable non-members" are in fact likely to be cluster members, with most of the mis-classified stars falling in the $30^{\prime}-40^{\prime}$ radial range. The effect of this potentially overly conservative membership selection on the calculated velocity dispersions will be discussed in the following section.

In Fig. 1 there are eight stars with velocities between 213 and $253 \mathrm{~km} \mathrm{~s}^{-1}$, plus stars 5_3_226 and 9_4_1918 which lie just outside the velocity interval, that have distances from the cluster center exceeding $46^{\prime}$. Only five of these ten stars survive the cluster membership analysis and are listed in Table 1 and shown as filled symbols in Fig. 5 . This number is too small for a statistically meaningful measure of the velocity dispersion at this extreme outer region and so the subsequent analysis will be based on the 155 probable $\omega$ Cen members that lie between $20^{\prime}$ and $46^{\prime}$ from the cluster center.

Before discussing the velocity dispersion profile defined by these data we show in Fig. 6 the surface density profile for $\omega$ Cen. The data are taken from DC08 (see references therein) except that we have used the surface density values determined from the cluster member sample derived here in place of the equivalent data in DC08. The surface density points from the inner and outer samples have been separately scaled vertically to match the existing data. The separate scaling is necessary since the outer sample covers a larger $V$ magnitude range than the inner sample. It is worth noting that the outer parts of this profile do not obviously exhibit the increased profile steepening at large radii characteristic of a tidally limited profile. Instead it appears that the outer profile is best described by a constant slope, i.e., a power-law profile. A (unweighted) least-squares fit to the data points lying beyond $20^{\prime}(1.3$ in $\log r$ ) yields a value of $-5.4 \pm 0.2$ for the power-law slope. The fit is shown as the straight line in the figure. It is also worth noting that the surface density implied by the 5 possible members beyond $45^{\prime}$, whose radial distances range between $47.4^{\prime}$ and $54.0^{\prime}$, is consistent with the observed profile and the power-law fit.

\section{The Velocity Dispersion Profile}

The outer sample of Sollima et al. (2009) contains $98 \omega$ Cen members that have distances from the cluster centre exceeding $\sim 20^{\prime}$, although there are only 13 members beyond $30^{\prime}$. Similarly, Scarpa \& Falomo (2010, see also Scarpa et al. (2003)) give radial velocities for $75 \omega$ Cen members with radial distances beyond $20^{\prime}$ from the cluster center, but there is only one member (just) 


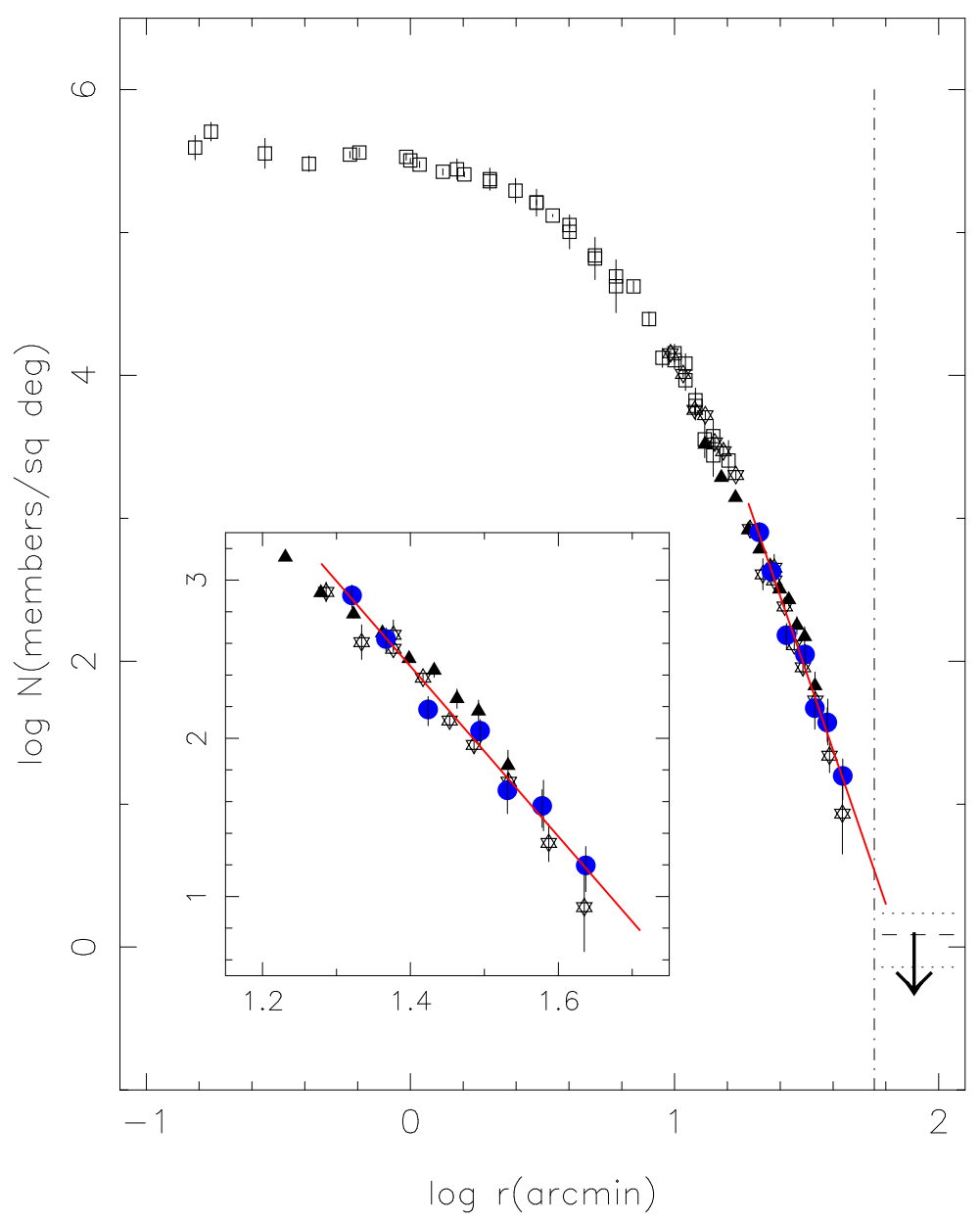

Fig. 6. - The (circularly averaged) surface density profile for $\omega$ Cen taken from Da Costa \& Coleman (2008) except that the blue filled circles, which represent the cluster member sample determined here, replace the equivalent data in the earlier work. The vertical dot-dash line indicates the tidal radius adopted by Da Costa \& Coleman (2008). The dashed line and the vertical arrow indicate the upper limit on the density of cluster members for the region between 1 and 2 tidal radii derived by Da Costa \& Coleman (2008), while the dotted lines represent the statistical uncertainty in the upper limit. The red solid line is a power law fit to the outer points; it has a slope of $-5.4 \mathrm{dex} / \mathrm{dex}$. The fit to the outer points is enlarged in the insert box. 
beyond $30^{\prime}$ in that sample. There are 26 stars in common between the Sollima et al. (2009) and Scarpa \& Falomo (2010) samples. The current sample, with 62 probable members between $30^{\prime}$ and $46^{\prime}$ therefore represents a considerable increase in the number of velocities with which to study the velocity dispersion profile in the extreme outer parts of this stellar system.

\subsection{AAT sample}

In Fig. 7 we show the velocity dispersion points calculated from the present sample of $\omega$ Cen probable members. The velocity dispersions have been calculated using a maximum likelihood estimator (e.g., Pryor \& Meylan 1993). In making the calculation the stars have been grouped into bins containing at least 10 members - the radial range of each bin and the number of stars included are indicated in the lower part of the figure. The dispersions have been calculated relative to the mean velocity for each group - these individual mean velocities differ by at most $2.3 \mathrm{~km} \mathrm{~s}^{-1}$ from the mean $\left(233.4 \mathrm{~km} \mathrm{~s}^{-1}\right.$ ) for the 93 members with distances between $20^{\prime}$ and $30^{\prime}$ from the cluster center. Calculating the dispersions for the stars beyond $30^{\prime}$ relative to this fixed mean causes only a slight increase ( 0.1 to $0.4 \mathrm{~km} \mathrm{~s}^{-1}$ ) in the dispersions but the differences are well within the uncertainties.

We also note that in the previous section it was suggested that the membership selection employed had been too conservative in that perhaps as many as a dozen of the "probable nonmembers" may be actual cluster members. To investigate the effect of this possibility on the calculated dispersions, we conducted five trials in which 12 stars were randomly selected from the set of 19 probable non-members that lie in the radial range $30^{\prime}$ to $46^{\prime}$. The selected stars were then combined with the probable members in the appropriate radial bins and the dispersions recalculated. In all cases the change in the dispersion was less than the errors calculated for the probable members only samples. The mean change was an increase in dispersion of $0.3 \mathrm{~km} \mathrm{~s}^{-1}$ with the largest excursions seen being an increase in the dispersion in the $33^{\prime}-36^{\prime}$ bin of $1.2 \mathrm{~km} \mathrm{~s}^{-1}$ and a decrease of $0.6 \mathrm{~km} \mathrm{~s}^{-1}$ in the dispersion for the $40^{\prime}-46^{\prime}$ bin. Both these changes are within the error for the equivalent probable members only sample. We conclude therefore that the dispersion measurements are stable against modest changes in the membership status of individual stars.

Shown also in Fig. 7 are the velocity dispersion measurements from Sollima et al. (2009), taken directly from their Table 1, from van de Ven et al. (2006) as the line-of-sight dispersions from their Figure 8, and from Scarpa \& Falomo (2010). In the latter case the dispersion points were calculated directly from the heliocentric radial velocities listed in Table 2 of Scarpa \& Falomo (2010) using identical techniques, including correction for perspective rotation, as those employed for the $\omega$ Cen stars observed here.

It is apparent from Fig. 7 that within the radial range where the different samples overlap, the velocity dispersion measures are consistent with one another. It is also evident that there is no evidence for any significant decline in the line-of-sight velocity dispersion of $\omega$ Cen members beyond 
$\sim 25^{\prime}$. In particular, the new data, which extend well beyond the previous data, are consistent with a constant line-of-sight velocity dispersion of $\sim 6.5 \mathrm{~km} \mathrm{~s}^{-1}$ in the outer parts of the cluster.

\subsection{Combined Sample}

The 26 stars in common between the Sollima et al. (2009) and the Scarpa \& Falomo (2010) samples have a mean velocity difference (Scarpa \& Falomo (2010) - Sollima et al. (2009)) of 0.2 $\mathrm{km} \mathrm{s}^{-1}$, with a standard deviation of $0.8 \mathrm{~km} \mathrm{~s}^{-1}$, confirming the velocity precision of both data sets. Unfortunately, due to the difference in the apparent magnitudes of the samples, there are no stars in common between the present work and that of Sollima et al. (2009). There are, however, three stars in the present sample that are also in Scarpa \& Falomo (2010). Scarpa \& Falomo (2010) stars 0006, 78004 and 85007 correspond to stars 8_8_4776,8_5_6453 and 7_4_160 here. For the first two stars the velocity differences (Scarpa \& Falomo (2010) - present work) are gratifyingly small: -0.6 and $-0.2 \mathrm{~km} \mathrm{~s}^{-1}$, respectively, but for the third star the Scarpa \& Falomo (2010) velocity exceeds that found here by $11.7 \mathrm{~km} \mathrm{~s}^{-1}$. Star 7_4_160 is one of the stars observed in all 12 configurations here and there is no indication of any velocity variability: the rms about the weighted mean velocity is $1.2 \mathrm{~km} \mathrm{~s}^{-1}$. Consequently, we have not used the Scarpa \& Falomo (2010) velocity for this star. Otherwise, the velocities for the stars in common have been averaged. The combined data set then has a total of 299 stars, 224 with radial distances between $20^{\prime}$ and $30^{\prime}$ and 75 lying between $30^{\prime}$ and $46^{\prime}$.

Figure 8 then shows the velocity dispersion profile for the combined sample. Shown also in the figure are velocity dispersion measurements calculated from the sample of Sollima et al. (2009) for stars between $12^{\prime}$ and $20^{\prime}$. The data are tabulated in Table 3. The line-of-sight velocity dispersion points from van de Ven et al. (2006) shown in Fig. 7 are also reproduced in the figure. The combined sample clearly verifies what was already evident from Fig. 7 - that although the velocity dispersion decreases outwards with increasing radius for the inner parts of the cluster (see, for example, figure 8 of Sollima et al. (2009) or figure 4 of Scarpa \& Falomo (2010)), beyond $\sim 25^{\prime}$ the velocity dispersion profile shows no signs of decreasing with increasing radius. This is despite, as noted above, the surface density dropping by a factor of $\sim 10$ between radial distances of $30^{\prime}$ and $40^{\prime}$. For the 140 stars in the combined sample with $r \geq 25^{\prime}$, the mean radius is $31.3^{\prime}$ and the velocity dispersion is $6.6 \pm 0.4 \mathrm{~km} \mathrm{~s}^{-1}$.

\section{Discussion}

The first question to be addressed is whether there is a dynamical model which can reproduce the surface brightness/surface density profile of the cluster, and the observed line-of-sight velocity dispersion profile, without any requirement for dark matter (i.e., a model in which mass follows light) or similarly, without any requirement for non-Newtonian gravity. For example, the Wilson (1975)- 


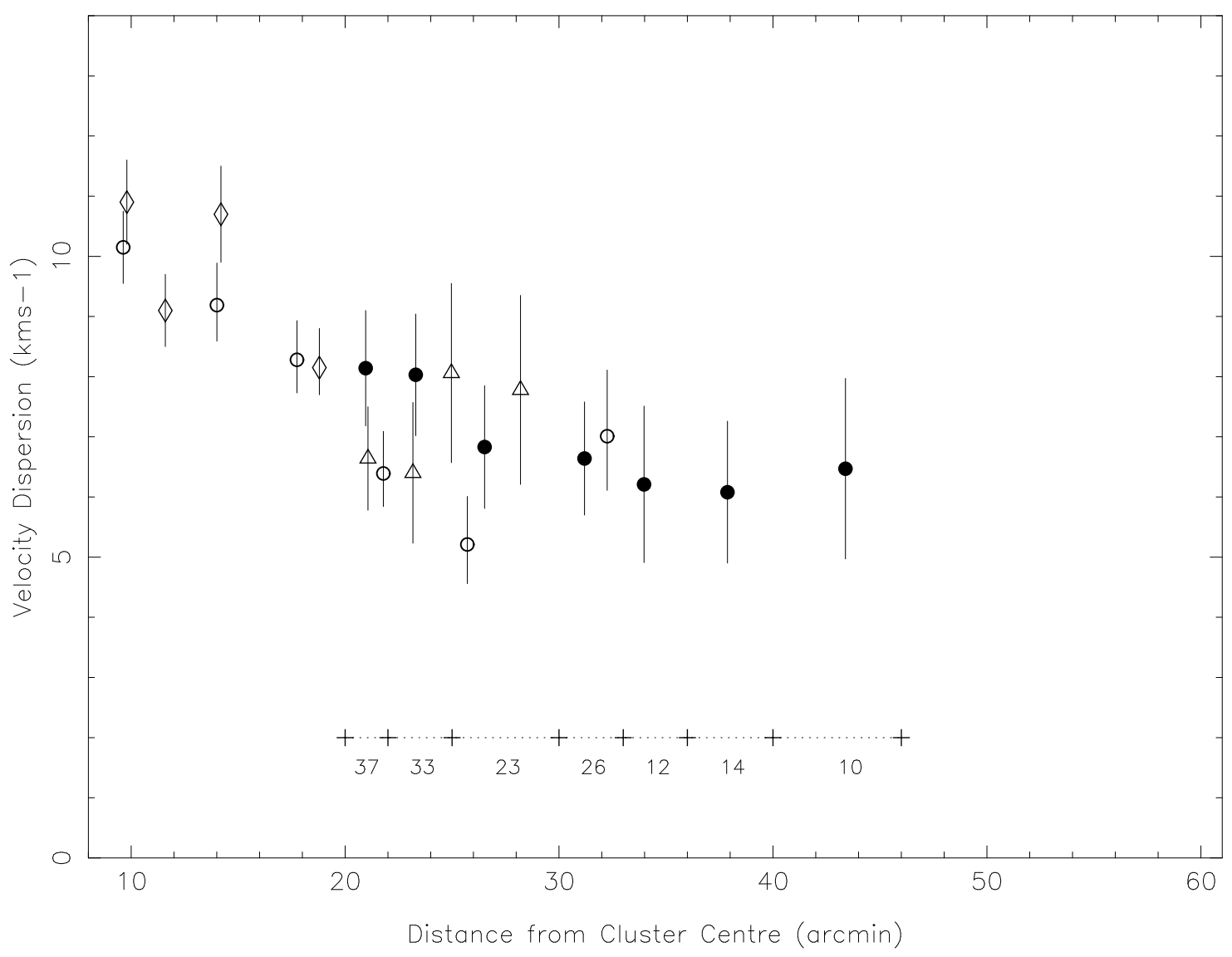

Fig. 7.- The line-of-sight velocity dispersion of $\omega$ Cen member stars is plotted against distance from the cluster center in arc minutes. The filled symbols are for the current probable members data set. The radial range corresponding to each dispersion point is indicated in the lower part of the plot as is the number of stars in each radius bin. Shown also on the plot are the line-ofsight velocity dispersion points of van de Ven et al. (2006) (open diamonds), Scarpa et al. (2003) (from Scarpa \& Falomo 2010, open triangles), and Sollima et al. (2009) (open circles). There is no evidence for any significant decline in the velocity dispersion beyond $\sim 25^{\prime}$. 
Table 3. Velocity dispersion profile for the outer parts of $\omega$ Cen from the combined sample

\begin{tabular}{ccccc}
\hline \hline $\begin{array}{c}\text { Radius Range } \\
(\text { arcmin })\end{array}$ & $\begin{array}{c}\text { Mean Radius } \\
(\text { arcmin })\end{array}$ & $\mathrm{N}$ & $\begin{array}{c}\sigma \\
\left(\mathrm{km} \mathrm{s}^{-1}\right)\end{array}$ & $\begin{array}{c}\text { error } \\
\left(\mathrm{km} \mathrm{s}^{-1}\right)\end{array}$ \\
\hline $12-14$ & 12.88 & 42 & 9.62 & 1.06 \\
$14-16$ & 15.12 & 44 & 8.85 & 0.95 \\
$16-18$ & 16.87 & 61 & 7.48 & 0.69 \\
$18-20$ & 18.98 & 39 & 8.49 & 0.97 \\
$20-22$ & 20.98 & 89 & 7.22 & 0.55 \\
$22-24$ & 23.08 & 55 & 7.92 & 0.77 \\
$24-27$ & 25.28 & 51 & 6.10 & 0.61 \\
$27-30$ & 28.55 & 29 & 7.74 & 1.03 \\
$30-33$ & 31.15 & 32 & 6.79 & 0.87 \\
$33-36$ & 34.14 & 15 & 6.03 & 1.12 \\
$36-40$ & 38.03 & 16 & 6.52 & 1.18 \\
$40-46$ & 43.35 & 12 & 6.22 & 1.32 \\
\hline
\end{tabular}

Note. - The first four entries are based on the data set of Sollima et al. (2009) while the remaining entries are drawn from the combined data set of this work, Sollima et al. (2009) and Scarpa \& Falomo (2010). 


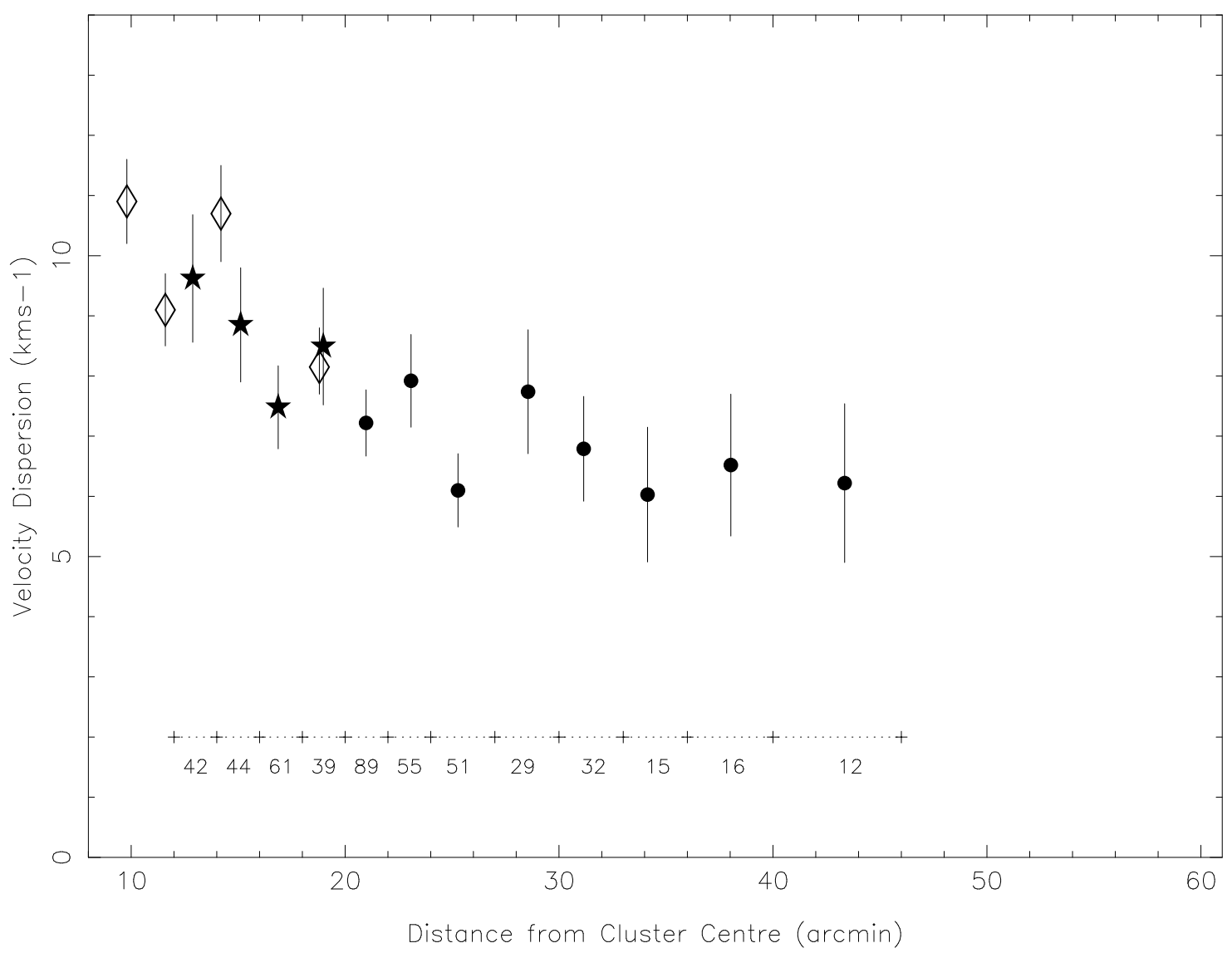

Fig. 8.- The line-of-sight velocity dispersion of $\omega$ Cen member stars is plotted against distance from the cluster center in arc minutes. The filled circles are for the combined data set of this work, Sollima et al. (2009), and Scarpa \& Falomo (2010). The filled stars are from the data set of Sollima et al. (2009) for stars between $12^{\prime}$ and 20'. Line-of-sight velocity dispersion points from van de Ven et al. (2006) are shown as open diamonds. For the combined and Sollima et al. (2009) points the radial range corresponding to each dispersion point is indicated in the lower part of the plot, as is the number of stars in each radius bin. As in Fig. 7, there is no evidence for any significant decline in the velocity dispersion beyond $\sim 25^{\prime}$. 
type model for $\omega$ Cen presented in Sollima et al. (2009) fits the projected surface density profile, the ellipticity profile and the rotation curve adequately, as shown in Figure 9 of Sollima et al. (2009). However, while the model also reproduces the Sollima et al. (2009) velocity dispersion data, it does not fit the more extensive velocity dispersion profile data presented here. The velocity dispersion profile of the model is consistently below the observed points in Fig. 8 beyond $\sim 25^{\prime}$, and declines monotonically to, for example, $3.6 \mathrm{~km} \mathrm{~s}^{-1}$ at $\sim 45^{\prime}$, significantly below the observations. The model is therefore not an adequate description of the dynamics in the outer parts of the cluster.

This is likely to be the case for all similar models, e.g., Mevlan et al. (1995); McLaughlin \& Mevlan (2003); McLaughlin \& van der Marel (2005)2, primarily because they are based on a fundamental assumption that the velocity distribution function $f(v)$ is of a 'lowered Maxwellian' form (King) 1966; Wilson 1975). This ensures that the density reaches zero at a finite radius, usually identified with the boundary set by the tidal force of the Milky Way (King 1966). In such models the velocity dispersion profile also declines monotonically, reaching zero at the same finite radius (e.g., Fig. 1 of Meylan et al. 1995). For $\omega$ Cen, however, the lack of an obvious tidal radius cutoff signature in the surface density profile (see Fig. 6) suggests that models of this type are not appropriate for the outer parts of the stellar system. In this sense the disagreement with the velocity dispersion observations reveals the inadequacy of the models, not necessarily anything more fundamental.

A more heuristic approach to the modelling of $\omega$ Cen is that taken by van de Marel \& Anderson (2010), whose primary aim was to place constraints on the possible presence of an intermediatemass black hole at the center of the cluster. Their approach was to parameterise the surface density profile and then solve the spherical anisotropic Jeans equation to predict the velocity dispersion profiles. The surface brightness profile was fit with a so-called "generalised nuker" profile (van de Marel \& Anderson 2010), which allows for a central power-law cusp and which contains two characteristic logarithmic slopes with associated "break" radii (see van de Marel \& Anderson 2010, for details). This adopted functional form does not have any particular physical significance, but in this context it is important to note that a density profile of this type, in contrast to the King (1966) and Wilson (1975)-type models described above, does not have a finite cutoff radius at which the density, and the velocity dispersion, go to zero.

We show in the left panel of Fig. 9 the best-fit "generalised nuker" model of van de Marel \& Anderson (2010) for the case where the central logarithmic slope $\gamma$ is fixed at zerd 3 , compared to the surface density data presented in Fig. 6. The model data have been scaled vertically to correspond to the

\footnotetext{
${ }^{2}$ The latter two references show a observed velocity dispersion point of $3.5 \pm 1.5 \mathrm{~km} \mathrm{~s}^{-1}$ at a radius of $36.6^{\prime}$, which is in accord with the predictions of the Wilson-type models. This datum comes from Seitzer (1983) and is based on velocities for four stars whose radial distances extend between $\sim 31^{\prime}$ and $\sim 44^{\prime}$. Three of the four stars are included in the sample of Sollima et al. (2009). We assert that the considerably more extensive data set presented here supercedes this early result.

${ }^{3}$ This model differs from the overall best-fit model, which has a shallow cusp with $\gamma=0.05$, only in the very inner regions of the cluster $\left(r \lesssim 20^{\prime \prime}\right)$.
} 
adopted surface density scale of the observations. The fit is excellent at all radii: in particular, the relatively constant logarithmic slope at large radii is in agreement with the observations, noting that the new surface density data presented here, and those given in DC08, were not included in the van de Marel \& Anderson (2010) fitting process. The right panel shows the corresponding line-of-sight velocity dispersion profile compared with the observations from Fig. 8 . The velocity distribution is mildly anisotropic with the transition from radial anisotropy at small radii to tangential anisotropy at large radii occurring at $r \approx 12^{\prime}$ (van de Marel \& Anderson 2010) in agreement with the results of van de Ven et al. (2006). The model velocity dispersion curve is consistent with the observations over the entire radial range depicted, including the points beyond $\sim 25^{\prime}$. For completeness we note that the upper panel of Figure 7 of van de Marel \& Anderson (2010) shows that this model also reproduces satisfactorily the observed velocity dispersion profile in the inner parts of the cluster. The model explicitly assumes 'mass follows light' and yields a $V$-band mass-to-light ratio of 2.6 in solar units, in agreement with the $\mathrm{M} / \mathrm{L}_{V}$ value found in the van de Ven et al. (2006) study. These dynamical values are entirely consistent with the mass-to-light ratio expected, given plausible assumptions about the stellar population of the cluster (e.g., Mevlan 1987). We can conclude therefore, based on this parameterized model, that an extended dark matter distribution is not required to reproduce the observed velocity dispersion profile in the outer parts of the cluster. Similarly, given that the modelling process is based on standard Newtonian dynamics through the use of the Jeans equation, no non-standard dynamics are required.

The van de Marel \& Anderson (2010) model may well be a reasonable description of the dynamics in the outer parts of $\omega$ Cen but because of its heuristic nature it does not provide any direct insight into the physical processes responsible for the applicability of the model. To investigate this we note again that the outer surface density profile of $\omega$ Cen is well represented by a powerlaw and does not exhibit the characteristic "King-profile" tidal cutoff seen in many clusters (e.g., McLaughlin \& van der Marel 2005; Jordi \& Grebel 2010). As discussed by Peñarrubia et al. (2009, see also Küpper et al. (2010)) this is an indication that the phase space in the outer parts of the cluster is likely populated up to $E \sim 0$, requiring a source of additional energy. The likely source of the required heating is the tidal shocks that occur each time $\omega$ Cen crosses the Galactic plane, as well as the tidal heating the cluster experiences as it moves in the spatially varying potential of the Galaxy.

Dinescu et al. (1999) used the current position and motion of $\omega$ Cen to characterise the orbit of the cluster around the Galactic Center. They found that the system has peri- and apoGalactocentric distances of approximately 1.2 and $6.2 \mathrm{kpc}$, and an orbital period of $\sim 120 \mathrm{Myr}$ (Dinescu et al. 1999). Using these orbital parameters van de Ven et al. (2006) calculate that the velocity component perpendicular to the Galactic plane $v_{\perp}$ is of order $40 \mathrm{~km} \mathrm{~s}^{-1}$. Consequently, for a disk scale height of $\sim 250 \mathrm{pc}$, it takes $\omega$ Cen about $12 \mathrm{Myr}$ to cross through the disk of the Galaxy (van de Ven et al. 2006). In contrast, in the $\omega$ Cen model of van de Ven et al. (2006) the orbital timescale for member stars in the outer parts of the cluster is approximately $100 \mathrm{Myr}$ at $r$ $\sim 25^{\prime}-30^{\prime}$, and longer at larger radii. Thus the impulse approximation (e.g., Gnedin et al. 1999; 

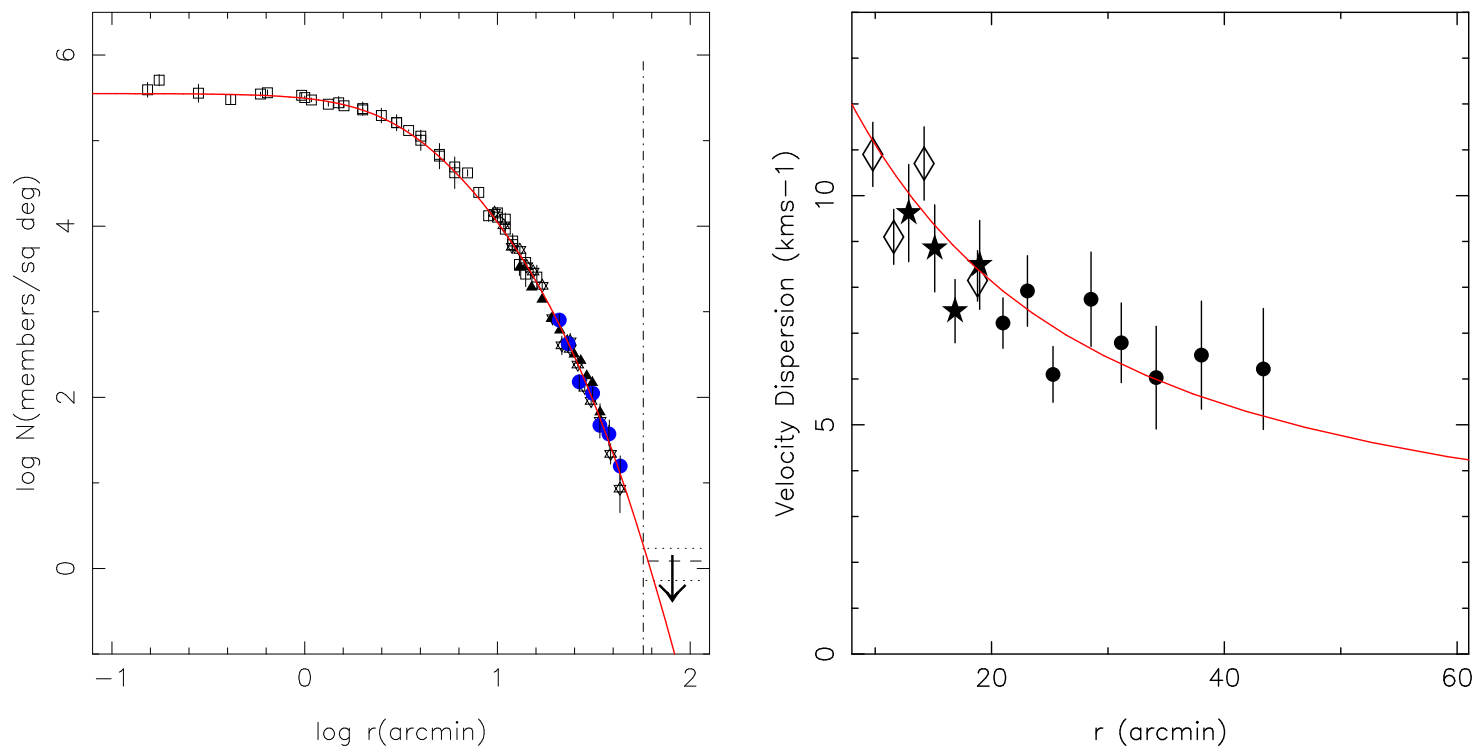

Fig. 9. - Left panel: The surface density profile for $\omega$ Cen, from Fig. 6. The red solid line is the surface density profile of the best-fit "generalised nuker" model of van de Marel \& Anderson (2010) for the case with the central logarithmic slope $\gamma$ fixed at zero. The model profile has been scaled vertically to fit the surface density observations. Right panel: The line-of-sight velocity dispersion of $\omega$ Cen for the outer parts of the cluster. Symbols are as for Fig. 8 . The red solid line is the lineof-sight velocity dispersion profile of the same "generalised nuker" model whose surface brightness profile is shown in the left panel. 
Binney \& Tremaine 1987, p. 446) is valid for calculating the "shock heating" the outer parts of the cluster experience each disk crossing.

We use equation 7-71 of Binney \& Tremaine (1987), with parameter values from van de Ven et al. (2006), to show that the impulsive change in the velocities of stars, $|\Delta v|$, is $\sim 0.36 r^{\prime} \mathrm{km} \mathrm{s}^{-1}$. This change is then comparable to, or exceeds, the line-of-sight velocity dispersion for radial distances beyond $r \approx 20^{\prime}$. The relative importance of disk shock heating is then measured by a comparison of the shock heating timescale $t_{\text {shock }}$ with the dynamical timescale $t_{\text {dun }}$. Use equation 7-72 of Binney \& Tremaine (1987) with the parameter values from van de Ven et al. (2006) gives:

$$
t_{\text {shock }}=475 \sigma^{2} / r^{2}
$$

for $t_{\text {shock }}$ in Myr, $\sigma$ in $\mathrm{km} \mathrm{s}^{-1}$ and $r$ in arcmint. Adopting $\sigma=6.5 \mathrm{~km} \mathrm{~s}^{-1}$ for $r \geq 25^{\prime}$ and then comparing the radial variation of $t_{\text {shock }}$ with that for $t_{d y n}$ from Figure 21 of van de Ven et al. (2006) shows that $t_{\text {shock }} \sim t_{\text {dyn }}$ at $r \sim 27^{\prime}$ and that $t_{\text {shock }}<t_{\text {dyn }}$ at larger radii. Consequently, we can conclude that beyond $r \sim 25-30^{\prime}$ the energy input to the outer parts of cluster from the disk shocking process is significant, and it will increasingly dominate the dynamics as the radius increases. Indeed when $t_{\text {shock }}$ is less than the stellar orbital timescale $\left(\sim 4 t_{d y n}\right.$, van de Ven et al. 2006) the stars are unlikely to be in equilibrium with the cluster potential. It is also worth noting that at approximately the same cluster radius as where shock heating becomes important, the stellar orbital timescale exceeds the orbital period of the cluster around the center of the Galaxy. As a result, the outer parts of the cluster will also experience tidal heating due to the changing potential field of the Galaxy as the cluster moves from its apo- to peri-Galactic distances (e.g., Gnedin \& Ostriker 1997). The significance of these two effects then suggests strongly that the phase space structure of the outer parts of $\omega$ Cen is dominated entirely by external effects driven by the cluster's location relatively close to the center of the Galaxy. The same conclusion was reached by van de Ven et al. (2006).

What is needed to shed further light on the situation is a full numerical simulation in which the dynamics of an $\omega$ Cen-like system are explored as the system orbits in the potential of the Galaxy. Such a calculation needs to include the effects of disk-shocking and continue for a sufficient time that the quasi-equilibrium situation that likely applies in the outskirts of $\omega$ Cen becomes established. The results of Küpper et al. (2010), for example, are suggestive in this respect. Based on $N$-body calculations of model star clusters with masses of a few $10^{4} \mathrm{M}_{\odot}$ on various orbits, Küpper et al. (2010) demonstrate that tidal heating can lead to a population of "potential escapers", i.e., energetically unbound stars inside the cluster's Jacobi radius. This then results in outer surface density profiles that have power law slopes in the range -4 to -5 . It also results in flattened velocity dispersion profiles that lie above the predictions of simple equilibrium models, with the

\footnotetext{
${ }^{4}$ The value of the numerical coefficient in this equation given by van de Ven et al. (2006), 21, is incorrect (G. van de Venn, priv. comm. 2012). However, the error does not significantly affect the discussion in van de Ven et al. (2006), although the influence of tidal shocks at a given radius are over-estimated in that paper.
} 
deviation commencing at about half of the Jacobi radius (Küpper et al. 2010). While Küpper et al. (2010) caution that their results are not readily scaleable to more massive globular clusters (recall the mass of $\omega$ Cen is a few $10^{6} \mathrm{M}_{\odot}$ ), their model calculations are at least qualitatively in agreement with the $\omega$ Cen observations.

More specific progress in this direction is given by the results of the N-body calculation described in Sollima et al. (2009). This is a $N=50,000$ particle model for the cluster, i.e., central concentration, tidal radius and mass similar to the real cluster, calculated for an $\omega$ Cen-like orbit in a three component (bulge+disk+halo) Galactic potential (see Sollima et al. (2009) for details). The calculations covered $\sim 10$ orbits (as against the many 10's of orbits made by the real cluster over a Hubble time). The velocity dispersion profile of the bound remnant at the end of the simulation (see Fig. 11 of Sollima et al. (2009)) is shown in Fig. 10. The agreement with the observations is excellent and it again suggests there is no need to invoke dark matter or non-Newtonian gravity to explain the observed velocity dispersion profile.

In summary then, the new observations presented here confirm that the velocity dispersion profile of $\omega$ Centauri remains relatively flat at $\sim 6.5 \mathrm{~km} \mathrm{~s}^{-1}$ beyond approximately $25^{\prime}$ from the cluster center. The most likely explanation of this effect is that we are seeing the consequences of external influence on the dynamics of the outer parts of the stellar system, which contain only a small fraction of the cluster stellar mass. Consequently, there is no requirement to invoke the presence of dark matter or non-standard gravitational theories to explain the observations.

GDaC would like to acknowledge the contributions of Dr. Matthew Coleman to the initial phases of this work, as well as the partial research support provided through Australian Research Council Discovery Projects grants DP0878137 and DP120101237. Useful conversations with Dr. Agris Kalnajs and Prof. Ken Freeman are also gratefully acknowledged as are the comments of the referee. The provision of unpublished model details by Dr. Roeland van der Marel and Dr. Antonio Sollima is also greatly appreciated, as is the excellent support at the telescope provided by the AAT support astronomers and night assistants.

Facilities: AAT (AAOmega)

\section{REFERENCES}

Armandroff, T. E., \& Da Costa, G. S. 1991, AJ, 101, 1329

Baumgardt, H., Grebel, E. K., \& Kroupa, P. 2005, MNRAS, 359, L1

Binney, J., \& Tremaine, S. 1987, Galactic Dynamics (Princeton, NJ: Princeton University Press)

Bekki, K., \& Freeman, K. C. 2003, MNRAS, 346, L11

Bellini, A., Bedin, A., Piotto, G., Milone, A. P., Marino, A. F., \& Villanova, S. 2010, AJ, 140, 631 


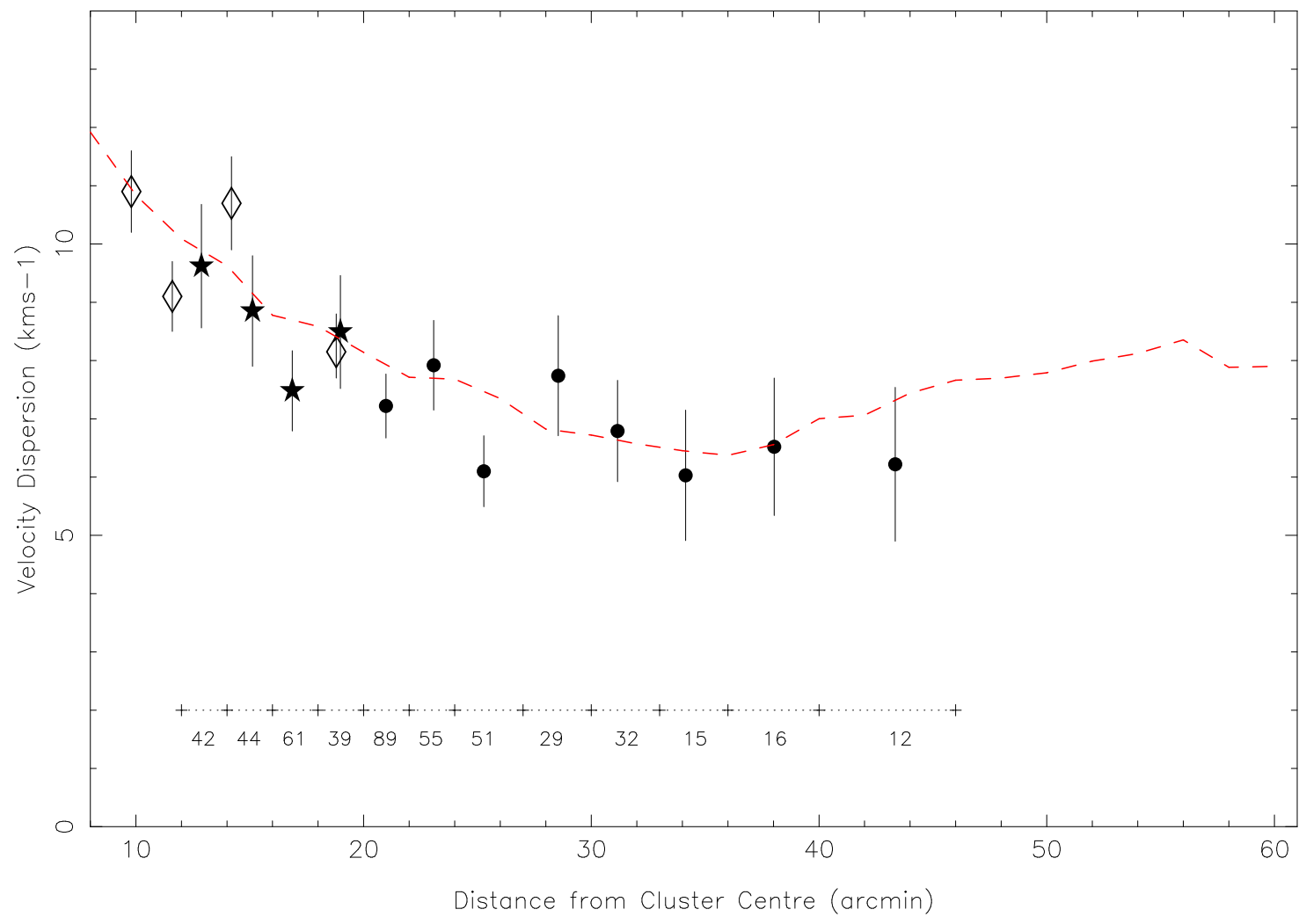

Fig. 10.- The line-of-sight velocity dispersion of $\omega$ Cen member stars is plotted against distance from the cluster center in arc minutes. Symbols are as for Fig. 8. Also shown as the red dashed line is the velocity dispersion profile of the $N$-body model described in Sollima et al. (2009) at the end of the simulation. 
Carraro, G., \& Lia, C. 2000, A\&A, 357, 977

Da Costa, G. S., \& Coleman, M. G. 2008, AJ, 136, 506 (DC08)

Dinescu, D. I., Girard, T. M., \& van Altena, W. F. 1999, AJ, 117, 1792

Dotter, A., Cahboyer, B., Jevremović, D., Kostov, V., Baron, E., Ferguson, J. W. 2008, ApJS, 178, 89

Freeman, K. C. 1993, in ASP Conf. Ser. 48, The Globular Cluster - Galaxy Connection, ed. G. H. Smith \& J. P. Brodie (San Francisco, CA: ASP), 608

Giersz, M., \& Heggie, D. C. 2003, MNRAS, 339, 486

Gnedin, O. Y., \& Ostriker, J. P. 1997, ApJ, 474223

Gnedin, O. Y., Lee, H. M., \& Ostriker, J. P. 1999, ApJ, 522, 935

Harris, W. E. 1996, AJ, 112, 1487 (H10)

Johnson, C. I., \& Pilachowski, C. A. 2010, ApJ, 722, 1373

Jordi, K., \& Grebel, E. K. 2010, A\&A, 522, A71

King, I. R. 1966, AJ, 71, 64

Küpper, A. H. W., Kroupa, P., Baumgardt, H., \& Heggie, D. C. 2010, MNRAS, 407, 2241

Lane, R. R., Kiss, L. L., Lewis, G. F., Ibata, R. A., Seibert, A., Bedding, T. R., \& Székely, P. 2009, MNRAS, 400, 917

Law, D. R., Majewski, S. R., Skrutskie, M. F., Carpenter, J. M., \& Ayub, H. F. 2003, AJ, 126, 1871

Majewski, S. R., Nidever, D. L., Smith, V. V., Damke, G. J., Kunkel, W. E., Patterson, R. J., Bizyaev, D., \& García Pérez, A. E. 2012, ApJ, 747, L37

Mashchenko, S., \& Sills, A. 2005, ApJ, 619, 243

McLaughlin, D. E., \& Meylan, G. 2003, in ASP Conf. Ser. 296, New Horizons in Globular Cluster Astronomy, ed. G. Piotto, G. Meylan, S. G. Djorgovski, \& M. Riello (San Francisco, CA: ASP), 153

McLaughlin, D. E., \& van der Marel, R. P. 2005, ApJS, 161, 304

Merritt, D., Meylan, G., \& Mayor, M. 1997, AJ, 114, 1074

Meylan, G. 1987, A\&A, 184, 144 
Meylan, G., Mayor, M., Duquennoy, A., \& Dubath, P. 1995, A\&A, 303, 761

Norris, J. E. 2004, ApJ, 612, L25

Norris, J. E., Freeman, K. C., \& Mighell, K. J. 1996, ApJ, 462, 241

Norris, J. E., Freeman, K. C.,Mayor, M., \& Seitzer, P. 1997, ApJ, 487, L87

Pancino, E., Pasquini, L., Hill, V., Ferraro, F. R., \& Bellazzini, M. 2002, ApJ, 568, L101

Pancino, E., Galfo, A., Ferraro, F. R., \& Bellazzini, M. 2007, ApJ, 661, L155

Pancino, E., Mucciarelli, A., Sbordone, L., Bellazzini, M., Pasquini, L., Monaco, L., \& Ferraro, F. R. 2011, A\&A, 527, A18

Peñarrubia, J., Navarro, J. F., McConnachie, A. W., \& Martin, N. F. 2009, ApJ, 698, 222

Piotto, G., Villanova, S., Bedin, L. R., Gratton, R., Cassisi, S., Momany, Y., Recio-Blanco, A., Lucatello, S., Anderson, J., King, I. R., Pietrinferni, A., \& Carraro, G. 2005, ApJ, 621, 777

Pryor, C., \& Meylan, G. 1993, in ASP Conf. Ser. 50, Structure and Dynamics of Globular Clusters, ed. S. Djorgovski \& G. Meylan (San Francisco, CA: ASP), 357

Robin, A. C., Reylé, C., Derrière, S., \& Picard, S. 2003, A\&A, 409, 523

Saunders, W., Gillingham, P., Haynes, R., Smith, G. A., Whittard, J. D., Churilov, V., Lankshear, A., Croom, S., Bridges, T., Jones, D., \& Boshuizen, C. 2004, Proc. SPIE, 5492, 389

Scarpa, R., \& Falomo, R. 2010, A\&A, 523, A43

Scarpa, R., Marconi, G., \& Gilmozzi, R. 2003, A\&A, 405, L15

Seitzer, P. O. 1983, Ph. D. thesis, Univ. Virginia

Sharp, R., Saunders, W., Smith, G., Churilov, V., Correll, D., Dawson, J., Farrel, T., Frost, G., Haynes, R., Heald, R., Lankshear, A., Mayfield, D., Waller, L., \& Whittard, D. 2006, Proc. SPIE, 6269, 62690G

Sollima, A., Bellazzini, M., Smart, R. L., Correnti, M., Pancino, E., Ferraro, F. R., \& Romano, D. MNRAS, 396, 2183

van de Marel, R. P., \& Anderson, J. 2010, ApJ, 710, 1063

van de Ven, G., van den Bosch, R. C. E., Verolme, E. K., \& de Zeeuw, P. T. 2006, A\&A, 445, 513

Wilson, C. P. 1975, AJ, 80, 175

Wylie-de Boer, E., Freeman, K., \& Williams, M. 2010, AJ, 139, 636

This preprint was prepared with the AAS LATEX macros v5.2. 